\title{
MATHEMATICAL MODELLING OF IMPURITY DIFFUSION IN GRAIN BOUNDARY NEIGHBORHOOD IN A DISLOCATION WALL FORCE FIELD (MODIFIED FISHER MODEL)
}

\author{
M.M. CHUIKO, A.K. FEDOTOV, V.G. RYCHAGOV \\ Institute of Mathematics \\ Surganova 11, Minsk 220072, Belarus \\ Belorussian State University \\ F.Skaryna av.4, Minsk 220050, Belarus \\ E-mail:dns@im.ba-net.by fedotov@bound.bsu.minsk.by
}

\begin{abstract}
In this work a computer simulation of impurity diffusion in the vicinity of individual bicrystalline grain boundary has been carried out with the use of a modified Fisher model in which the grain's boundary influence (in the form of internal mechanical stresses) onto impurity atoms is taken into account. In computations the monotone difference schemes of the second order of accuracy on irregular grids were used. For such schemes under intrinsic restrictions on the grid steps the stability in the uniform norm has been proved.
\end{abstract}

\section{INTRODUCTION}

Grain Boundaries (GBs) affects most of electronic, strengthening and other properties of polycrystals. Their influence is mainly due to more active mass transfer by GBs than in grains. Hence a knowledge of impurity profiles in the vicinity of GBs is required to understand physical processes which are controlled by diffusion. Most descriptions of GB diffusion up till now have been based upon the known Fisher model for bicrystals (see, [1]). According to this model, GB is defined as semi-infinite isotropic layer (confined by two semi-infinite grains with a lower diffusivity $D_{g}$ ) of uniform thickness $\delta$ with high diffusivity $D_{b}, D_{b}>>D_{g}$. GB plane is normal to the interface bicrystal/source through which diffusant with concentration co is transferred. However the Fisher model can be rather far from reality because it fails to 


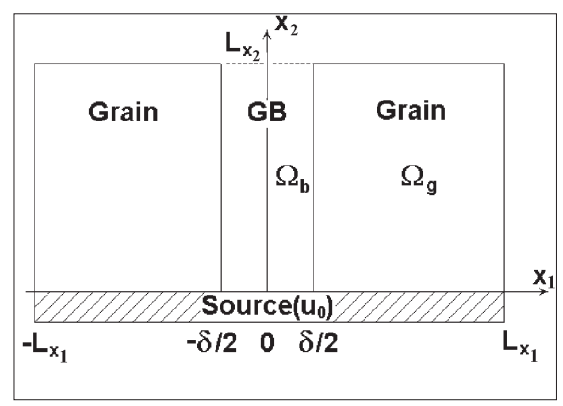

Figure 1. Calculated region of a problem

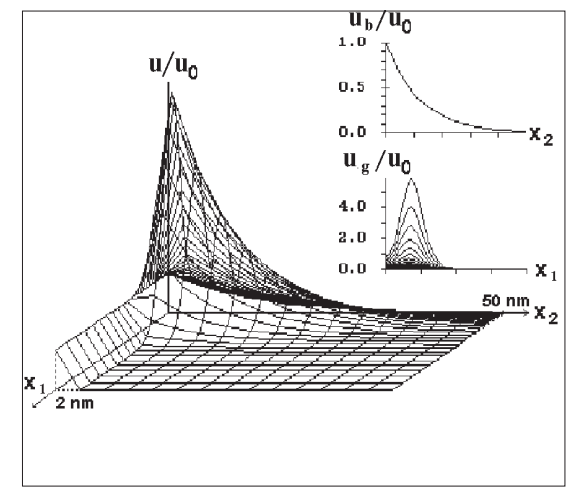

Figure 2. 3D and 2D pictures for $S b$ distribution in the vicinity of the $G e<S b>$ bicrystalline $G B$

account for the influence of added forces acting on impurity atoms from GB (elastic, electric, etc.). In this work a computer simulation of impurity diffusion in the vicinity of bicrystalline GB has been carried out with the use of a modified Fisher model taking into account the effect contributed by the internal mechanical stresses acting on impurity atoms from GB. In this case GB "core" was treated as a dislocation wall [2] acting on impurity atoms with force $F\left(x_{1}\right)=F_{0} \exp \left[-2 \pi\left(x_{1}-\delta / 2\right) / h_{d s}\right]$ in the direction $x_{1}$ normal to GB plane. Pre-factor $F_{0}=4 \pi^{2} \varepsilon r^{3} G b(1+\nu) / 3 h_{d s}^{2}(1-\nu)$ is determined by a relative expansion (compression) of matrix lattice due to impurity atoms $\varepsilon=\left(r_{i}-r_{m}\right) / r_{i}\left(r_{i}\right.$ and $r_{m}$ are atomic covalent radii for impurity and matrix atoms respectively), shear modulus $G$, Poisson coefficient $\nu$, Burgers vector $b$ and distance $h_{d s}$ between dislocations.

\section{PROBLEM DEFINITION AND DIFFERENCE SCHEME}

Impurity diffusion problem can be formulated as a conjunction problem in the rectangle $\bar{\Omega}=\bar{\Omega}_{b} \bigcup \bar{\Omega}_{g}$ (see Fig.1),

$$
\begin{gathered}
\Omega_{b}=\left\{\left(x_{1}, x_{2}\right), \quad 0<x_{1}<\delta / 2, \quad 0<x_{2}<L_{x_{2}}\right\}, \\
\Omega_{g}=\left\{\left(x_{1}, x_{2}\right), \quad \delta / 2<x_{1}<L_{x_{1}}, \quad 0<x_{2}<L_{x_{2}}\right\} .
\end{gathered}
$$

In the domain $\Omega_{b}$ we shall find a solution of the equation

$$
\frac{\partial u_{b}}{\partial t}=D_{b}\left(\frac{\partial^{2} u_{b}}{\partial x_{1}^{2}}+\frac{\partial^{2} u_{b}}{\partial x_{2}^{2}}\right), \quad\left(x_{1}, x_{2}, t\right) \in \Omega_{b} \times\left(0, t_{0}\right),
$$


and in the domain $\Omega_{g}$ the following one

$$
\frac{\partial u_{g}}{\partial t}=D_{g} \frac{\partial^{2} u_{g}}{\partial x_{1}^{2}}-\frac{\partial}{\partial x_{1}}\left(v\left(x_{1}\right) u_{g}\right), \quad\left(x_{1}, x_{2}, t\right) \in \Omega_{g} \times\left(0, t_{0}\right) .
$$

The continuity conditions for the solution

$$
\left.u_{b}\left(x_{1}, x_{2}, t\right)\right|_{x_{1}=\delta / 2-0}=\left.u_{g}\left(x_{1}, x_{2}, t\right)\right|_{x_{1}=\delta / 2+0}
$$

and for the flow

$$
\left.D_{b} \frac{\partial u_{b}}{\partial x_{1}}\right|_{x_{1}=\delta / 2-0}=\left.\left(D_{g} \frac{\partial u_{g}}{\partial x_{1}}-v u_{g}\right)\right|_{x_{1}=\delta / 2+0}
$$

are satisfied at the conjunction line $x_{1}=\delta / 2$. At the boundary of the domain $\Omega$ the following boundary conditions are given

$$
\begin{array}{r}
\left.\frac{\partial u_{b}}{\partial x_{1}}\right|_{x_{1}=0}=0,\left.u_{g}\right|_{x_{1}=L_{x_{1}}}=0, \\
\left.u_{b}\right|_{x_{2}=0}=\left.u_{g}\right|_{x_{2}=0}=u_{0},\left.u_{b}\right|_{x_{2}=L_{x_{2}}}=\left.u_{g}\right|_{x_{2}=L_{x_{2}}}=0 .
\end{array}
$$

Initial data has the form

$$
\left.u\right|_{t=0}= \begin{cases}u_{0}, & x_{2}=0 \\ 0, & x_{2} \neq 0\end{cases}
$$

where $u=u_{b}$ or $u=u_{g}$.

Further, for the function $v\left(x_{1}\right)$ we shall suppose a fulfillment of inequality $v\left(x_{1}\right) \geq 0, \forall x_{1} \in \Omega$. From the physical point of view it describes the situation when the force acting on impurity atoms is directed outside the domain $\Omega$. In general, the function $v\left(x_{1}\right)$ has a form $v\left(x_{1}\right)=D_{g} F\left(x_{1}\right) / k T$.

We shall find a solution of the problem (2.1)-(2.6) using the difference schemes of the second order of approximation on irregular grids [4]-[6]. Let us suppose that in the problem's domain of definition $\bar{\Omega} \times\left[0, t_{0}\right]$ we have a grid $\bar{\omega}_{h \tau}=\hat{\bar{\omega}}_{h} \times \bar{\omega}_{\tau}$, where $\bar{\omega}_{\tau}=\left\{t_{j}=j \tau, j=0,1, \ldots, j_{0}, j_{0} \tau=t_{0}\right\}$, $\hat{\bar{\omega}}_{h}=\hat{\bar{\omega}}_{1} \times \omega_{2}, \hat{\bar{\omega}}_{1}=\left\{x_{1 m+1}=x_{1 m}+h_{1 m}, m=\overline{1, M}, x_{0}=0, x_{M}=L_{x_{1}}\right\}$, $\bar{\omega}_{2}=\left\{x_{2 n}=n h_{2}, n=\overline{0, N}, h_{2 n}=L_{x_{2}} / N\right\}$. Thus, in the direction $x_{1}$ we use an irregular grid and in the direction $x_{2}$ a uniform one.

Let us note that the selection of a uniform in the direction $x_{2}$ grid is determined by the model's specific character. The diffusion process is supposed flowing very slow in the direction $x_{2}$. As a result it can be disregarded. The selection of the irregular grid is determined by the fact of small geometric 
measures of the domain $\Omega_{b}$ in which a convective transfer in the direction $x_{1}$ takes place.

On the grid $\bar{\omega}_{h \tau}$ let us approximate the problem (2.1)-(2.6) by the following difference scheme

$$
\begin{gathered}
y_{t}-\frac{2 D_{b}}{h_{1}} y_{x_{1}}=D_{b} y_{\bar{x}_{2} x_{2}}, \quad m=0 \\
\left(y+\delta_{1 m} y_{\bar{x}_{1}}+\delta_{2 m} y_{x_{1}}\right)_{t}=D_{b}\left(\hat{y}_{\bar{x}_{1} \hat{x}_{1}}\right. \\
\left.+\left(y+\delta_{1 m} y_{\bar{x}_{1}}+\delta_{2 m} y_{x_{1}}\right)_{\bar{x}_{2} x_{2}}\right), \quad m=\overline{1, m_{0}-1}, \\
\frac{\kappa_{m}+1}{2} y_{t}=\frac{\kappa_{m}}{h_{1 m}}\left[D_{g} \hat{y}_{x_{1}}-D_{b} \hat{y}_{\bar{x}_{1}}\right]+\frac{1}{2}\left[r^{+} \hat{y}_{x_{1}}+r^{-} \hat{y}_{\bar{x}_{1}}\right] \\
-\left[\frac{d_{m}}{2}-\frac{r_{m} \kappa_{m}}{h_{1 m}}\right] \hat{y}_{m}+\frac{\kappa_{m}}{2} D_{b} y_{\bar{x}_{2} x_{2}}, \quad m=m_{0}, \\
\left(y+\delta_{1 m} y_{\bar{x}_{1}}+\delta_{2 m} y_{x_{1}}\right)_{t}=\kappa_{m} D_{g} \hat{y}_{\bar{x}_{1} \hat{x}_{1}}+\bar{r}_{m}^{+} \hat{y}_{x_{1}}+\bar{r}_{m}^{-} \hat{y}_{\bar{x}_{1}} \\
-d_{m}\left(\hat{y}+\delta_{1 m} \hat{y}_{\bar{x}_{1}}+\delta_{2 m} \hat{y}_{x_{1}}\right), \quad m=\bar{m}_{0}+1, M, \\
\hat{y}_{m 0}=u_{0}, \hat{y}_{m N}=0, m=\hat{y}_{M n}=0, n=\hat{y}_{1, N-1}, \\
\hat{y}_{M n},
\end{gathered}
$$

where

$$
\begin{aligned}
\kappa_{m} & =\frac{1}{1+R_{m}}, \\
R_{m} & =\frac{\bar{r}_{m}^{+}}{D_{g}} \frac{h_{1 m+1}+2 h_{1 m}}{6}-\frac{\bar{r}_{m}^{-}}{D_{g}} \frac{h_{1 m}+2 h_{1 m+1}}{6} \\
\bar{r}_{m}^{ \pm} & =0,5\left(\bar{r}_{m} \pm\left|\bar{r}_{m}\right|\right) \\
\bar{r}_{m} & =-v\left(\bar{x}_{1_{m}}\right), \quad \bar{x}_{1_{m}}=x_{1 m}+\tilde{h}_{m} \\
\tilde{h}_{m} & =\frac{h_{1 m+1}-h_{1 m}}{3}, \quad d_{m}=v^{\prime}\left(\bar{x}_{1_{m}}\right) \\
\delta_{1 m} & =\tilde{h}_{m}^{-}, \quad \delta_{2 m}=\tilde{h}_{m}^{+}, \quad \tilde{h}_{m}^{ \pm}=0,5\left(\tilde{h}_{m} \pm\left|\tilde{h}_{m}\right|\right) .
\end{aligned}
$$

Here we suppose that the conjunction line $x_{1}=\delta / 2$ hits a node $x_{1}=x_{1 m_{0}}$ of the grid $\hat{\bar{\omega}}_{h}$ and $h_{1 m_{0}-1}=h_{1 m_{0}}$, i.e. in the vicinity of $x_{1}=\delta / 2$ the grid is uniform. It is easy to show from results of [4] - [6] that the scheme 
approximates the problem (2.1)-(2.6) with the second local order at point $\left(\bar{x}_{1_{m}}, x_{2 n}, t_{j}\right)$ in subdomains $\Omega_{b}$ and $\Omega_{g}$. Note that conjunction conditions are approximated with use of the equation such that the order of approximation also equals to two.

Taking into account that the difference equations can be written in the form

$$
A_{m n} y_{m-1 n}-C_{m n} y_{m n}+B_{m n} y_{m+1 n}=-F_{m n},
$$

where

$$
\begin{aligned}
& A_{m n}= \begin{cases}0, & m=0, \\
\frac{\tau D_{b}}{\hbar_{1 m} h_{1 m}}+\frac{\delta_{1 m}}{h_{1 m}}, & m=\overline{1, m_{0}-1,} \\
\frac{\tau \kappa_{m} D_{b}}{h_{1 m}^{2}}-\frac{\tau r_{m}^{-}}{2 h_{1 m}}, & m=m_{0}, \\
\frac{\tau \kappa_{m} D_{g}}{\hbar_{1 m} h_{1 m}}-\frac{\tau \bar{r}_{m}^{-}}{h_{1 m}}+\frac{\delta_{1 m}}{h_{1 m}}\left(1+\tau d_{m}\right), & m=\overline{m_{0}+1, M-1,}\end{cases} \\
& B_{m n}= \begin{cases}\frac{2 \tau D_{b}}{h_{1 m+1}^{2}}, & m=0, \\
\frac{\tau D_{b}}{\hbar_{1 m} h_{1 m+1}}-\frac{\delta_{2 m}}{h_{1 m+1}}, & m=\overline{1, m_{0}-1,} \\
\frac{\tau \kappa_{m} D_{g}}{h_{1 m}^{2}}+\frac{\tau r_{m}^{+}}{2 h_{1 m}}, & m=m_{0}, \\
\frac{\tau \kappa_{m} D_{g}}{\hbar_{1 m} h_{1 m+1}}+\frac{\tau \bar{r}_{m}^{+}}{h_{1 m+1}}-\frac{\delta_{2 m}}{h_{1 m+1}}\left(1+\tau d_{m}\right), & m=\overline{m_{0}+1, M-1},\end{cases} \\
& C_{m n}= \begin{cases}A_{m n}+B_{m n}+1, & m=\overline{0, m_{0}-1}, \\
A_{m n}+B_{m n}+\frac{1}{2}\left(\kappa_{m}+1+\tau d_{m}\right)-\frac{\tau r_{m} \kappa_{m}}{h_{1 m}}, & m=m_{0}, \\
A_{m n}+B_{m n}+1+\tau d_{m}, & m=\overline{m_{0}+1, M-1,}\end{cases} \\
& F_{m n}= \begin{cases}y_{0 n}+\tau D_{b} y_{\bar{x}_{2} x_{2}, 0 n}, & m=0, \\
y_{\left(\omega_{1}, \omega_{2}\right) m n}+\tau D_{b} y_{\left(\omega_{1}, \omega_{2}\right) \bar{x}_{2} x_{2} m n}, & m=\overline{1, m_{0}-1}, \\
\frac{\kappa_{m}+1}{2} y_{m n}+\frac{\tau \kappa_{m}}{2} D_{b} y_{\bar{x}_{2} x_{2} m n}, & m=m_{0}, \\
y_{\left(\omega_{1}, \omega_{2}\right) m n}, & m=\overline{m_{0}+1, M-1},\end{cases} \\
& y_{\left(\omega_{1}, \omega_{2}\right) m n}=\left(y+\delta_{1} y_{\bar{x}_{1}}+\delta_{2} y_{x_{1}}\right)_{m n} \\
& =\omega_{1 m} y_{m+1 n}+\left(1-\omega_{1 m}-\omega_{2 m}\right) y_{m n} \\
& +\omega_{2 m} y_{m-1 n} \text {, }
\end{aligned}
$$




$$
\omega_{1 m}=\frac{\delta_{2 m}}{h_{m+1}}, \quad \omega_{2 m}=-\frac{\delta_{1 m}}{h_{m}},
$$

we solve the problem by the difference sweep method. At $m=0$ for uniformity we suppose that $A_{m n}=0$. A stencil of the scheme at $x_{1}=0$ consists of two nodes $\left(x_{10}, x_{2 n}\right)$ and $\left(x_{11}, x_{2 n}\right)$ and a value of $A_{0 n}$ does not defined, in general. Applying the maximum principle [3] we shall take this circumstance into account.

A sufficient condition of positivity of coefficients of (2.9) can be formulated in the form of inequality $\tau>c\left(h_{1 m+1}^{2}-h_{1 m}^{2}\right)$ From the form of coefficients it follows that the diagonal dominance carries out when $\tau<0,5\left|d_{m}\right|^{-1}$. Additional restriction on step $\tau$ that provides stability of the difference scheme is resulted from the explicit approximation of derivative in direction $x_{2}$ and has a form $\tau<c h_{2}^{2} / D_{b}$.

In order to investigate the stability of the difference scheme (2.7) let us apply a consequence of the maximum principle ([3]).

Let $y=y(P)$ be a solution of the heterogeneous equation

$$
\begin{aligned}
& L(P) \equiv A(P) y(P)-\sum_{Q \in \mathcal{S}^{\prime}(P)} B(P, Q) y(Q)=F(P) \text { on } \omega, \\
& y(P)=0 \text { on } \gamma
\end{aligned}
$$

Here $P$ is a point of the stencil $\mathcal{S}(P), \mathcal{S}^{\prime}(P)$ is a vicinity of the node $P$.

Theorem 2.1. If $D(P) \equiv A(P)-\sum_{Q \in \mathcal{S}^{\prime}(P)} B(P, Q)>0$ everywhere on $\omega$ then for solution of the problem (2.10) we have the following estimate

$$
\|y\|_{C} \leq\left\|\frac{F}{D}\right\|_{C} .
$$

Let us suppose that $u_{0}=0$ in (2.5). Then

$$
\begin{aligned}
D_{m n} & =C_{m n}-A_{m n}-B_{m n} \\
& = \begin{cases}1, & m=\overline{0, m_{0}-1}, \\
\frac{1}{2}\left(\kappa_{m}+1+\tau d_{m}\right)-\frac{\tau r_{m} \kappa_{m}}{h_{1 m}}, & m=m_{0}, \\
1+\tau d_{m}, & m=\overline{m_{0}+1, M-1},\end{cases}
\end{aligned}
$$

It is easy to see that $\left\|D^{-1}\right\|_{C} \leq\left\|\frac{1}{1-\tau|d|}\right\|_{C} \leq 1+2 \tau\|v\|_{C}$. To estimate the right side we shall consider two cases. First we shall show that for any function $v_{m n}$ on the grid $\hat{\omega}_{h}$ the following inequality takes place

$$
\left|v_{\left(\omega_{1}, \omega_{2}\right), m n}\right| \leq\|v\|_{C}
$$


Indeed, $\left|v_{\left(\omega_{1}, \omega_{2}\right), m n}\right|=\left|\omega_{1 m} v_{m+1 n}+\left(1-\omega_{1 m}-\omega_{2 m}\right) v_{m n}+\omega_{2 m} v_{m-1 n}\right| \leq$ $\left|\omega_{1 m}+\left(1-\omega_{1 m}-\omega_{2 m}\right)+\omega_{2 m}\right|\|v\|_{C}=\|v\|_{C}$. Taking into account that $\omega_{1 m} \geq 0$ and $\omega_{2 m} \geq 0$ we can see that the last inequality holds when $1-$ $\omega_{1 m}-\omega_{2 m} \geq 0$. But the last inequality is valid since for $h_{1 m+1}>h_{1 m}$ we have $\omega_{1 m}=\frac{\bar{h}_{1 m+1}-h_{1 m}}{3 h_{1 m+1}}, \omega_{2 m}=0$ and $1-\omega_{1 m}-\omega_{2 m}=\frac{2 h_{1 m+1}+h_{1 m}}{3 h_{1 m+1}}>0$. For $h_{1 m+1}<h_{1 m}$ we get $\omega_{1 m}=0, \omega_{2 m}=-\frac{h_{1 m+1}-h_{1 m}}{3 h_{1 m}}, 1-\omega_{1 m}-\omega_{2 m}=$ $\frac{h_{1 m+1}+2 h_{1 m}}{3 h_{1 m}}>0$.

Let us note that for any function $v$ on $\omega_{h \tau}$ the following inequality holds

$$
\left\|v+\tau c v_{\bar{x}_{2} x_{2}}\right\|_{C} \leq\|v\|_{C}
$$

when $\tau<h_{2}^{2} / c$.

Applying consequently (2.11) and (2.12) and taking into account that $\kappa_{m}<$ 1 , for the right side $F_{m n}$ we get

$$
\|F\|_{C} \leq\|y\|_{C}
$$

Thus, from Theorem 1 and estimates for $\|F\|_{C}$ and $\left\|D^{-1}\right\|_{C}$ it follows that

$$
\left\|y\left(t^{j+1}\right)\right\|_{C} \leq \rho\left\|y\left(t^{j}\right)\right\|_{C}, \quad \rho=1+2 \tau\|v\|_{C}
$$

The last estimate expresses the property of $\rho$ - stability of (2.7).

Let us note that requiring the condition $\tau<c \max _{m} h_{1 m}, c=0,5 /\|v\|_{C}$, we can avoid the restriction $v\left(x_{1}\right)>0$ and preserve the estimate (2.13).

\section{COMPUTING OF IMPURITY PROFILES IN THE VICINITY OF GRAIN BOUNDARIES}

As is seen from the modified Fisher model (2.1)-(2.6), a character of impurity distribution $u_{g}\left(x_{1}, x_{2}\right)$ around GB plane for constant time $t$ (which means either heat treatment time or time of bicrystal growing) will be determined by the sign of elastic force $F\left(x_{1}\right)$ acting on impurity from the boundary "core" and by the relation between diffusion and drift terms in (2.2). As literature data show (see, [1]), in the most known experiments on GB diffusion $D_{b}>D_{g}$. This actually allows (as in the standard Fisher model) the influence of impurity distribution along GB "core" $u_{b}\left(x_{2}\right)$ on the character of impurity distribution $u_{g}\left(x_{1}\right)$ in the vicinity of GB to be disregarded. From the calculations it is seen that the rate of impurity concentration growth in GB "core" decreases rapidly with time even at $D_{b} \geq 1.5 D_{g}$.

It means that the character of $u_{b}\left(x_{2}\right)$ for short and long times of diffusion $t$ is practically the same. That is the reason that as for usual Fisher model the 
"core" of bicrystalline GB can be treated as a source of diffusant with constant impurity concentration $u_{b}\left(x_{2}\right)$ for diffusion of impurity into the grain bulk. Thus, a deviation of real impurity profile $u_{g}\left(x_{1}, x_{2}\right)$ from Fisher distribution in the neighborhood of GB [1] will be determined mainly by the drift term in (2). In calculations presented here, all grain-bulk diffusivities $D_{g}$ were defined by the equation $\exp D^{(G)}=D_{0}^{(G)}\left(-Q^{(G)} / k T\right)$ on the basis of activation energies $Q^{(G)}$ and coefficients $D_{0}^{(G)}$ for impurities taken from [7].Covalent radii for crystal-matrices $(S i, G e)$ and impurities

$(S b, A l, B, O)$ were taken from [8]. Distances $h_{d s}$ between dislocations in real bicrystalline GBs can be estimated from measurements of the density of electrically active centers at GB plane. As is obvious from [9], the latter is usually varied over the range $1012-1014 \mathrm{~cm}^{-2}$. Since the number of "dangling" bonds along a unit dislocation (which just create these active centers) should not exceed $107 \mathrm{~cm}^{-1}$, the number of dislocations per $1 \mathrm{~cm}$ of GB length will not be higher than $105-107$. This means that the average interdislocation distance at GB plane can be varied within $10^{-9} \leq h_{d s} \leq 10^{-7} \mathrm{~m}$. The numerical calculations of impurity profiles in the neighborhood of bicrystalline GB were performed for the following matrix-impurity pairs: $G e<S b\rangle, S i<A l>$, $S i<O>$, Si $<B>$. For the first two pairs an elastic force is positive $\left(F_{0}>0\right)$ and it will force out impurity of GB "core" whereas for the last two pairs $F_{0}<0$ and impurity will be attracted to GB "core". When calculating, the ratios $D_{b} / D_{g}$ were taken as $1.5-105$ and the "thickness" $\delta$ of GB "core", in accordance with [1], was chosen as $10^{-9} \mathrm{~m}$. All calculations were performed for the temperature $1000 \mathrm{~K}$. The remaining values for calculations were taken from [8]: $r_{S i}=0.117 \mathrm{~nm}, r_{G e}=0.122 \mathrm{~nm}, r_{A l}=0.143 \mathrm{~nm}, r_{S b}=0.141 \mathrm{~nm}$, $r_{B}=0.089 \mathrm{~nm}, r_{O}=0.117 \mathrm{~nm} ; G_{S i}=1.1011 \mathrm{~N} / \mathrm{m}^{2}, G_{G e}=5.1011 \mathrm{~N} / \mathrm{m}^{2} ;$ $n_{S i}=0.25, n_{G e}=0.35 ; D_{g}^{(A l)}=3.648 \cdot 10^{-21} \mathrm{~m}^{2} / \mathrm{s}, D_{g}^{(S b)}=2.576 \cdot 10^{-16} \mathrm{~m}^{2} / \mathrm{s}$, $D_{g}^{(B)}=1.646 \cdot 10^{-22} \mathrm{~m}^{2} / \mathrm{s}, D_{g}^{(O)}=1.365 \cdot 10^{-15} \mathrm{~m}^{2} / \mathrm{s} ; b_{S i}=0.543 \mathrm{~nm}, b_{G e}=$ $0.566 \mathrm{~nm}$.
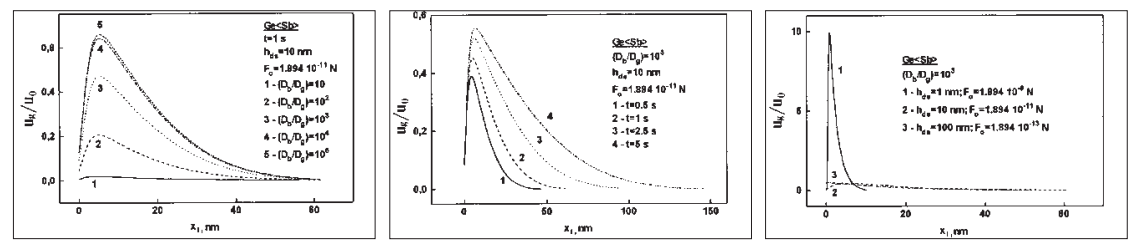

Figure 3. 3. $G e\langle S b\rangle$ impurity profiles $u_{g}\left(x_{1}\right)$ for different parameters calculated at depth of $35 \mathrm{~nm}$ from interface source/bicrystalc

A computer code developed to simulate diffusion in terms of a modified Fisher model enables the results of calculations to be presented as $3 D$ picture of impurity concentration distribution $u_{g}\left(x_{1}, x_{2}\right)$ around GB, normalized to the concentration of diffusant in a source $u_{0}$. Besides, the computer code makes it possible to derive from $3 D$ picture $2 D$ sections $u_{g}\left(x_{1}\right)$ and $u_{b}\left(x_{2}\right)$ for 

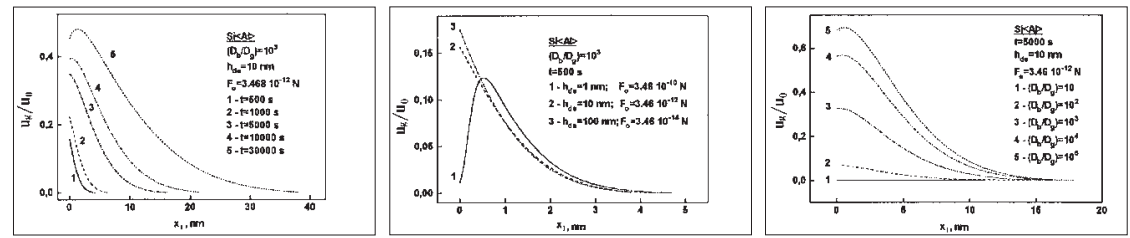

Figure 4. 4. $S i\langle A l\rangle$ impurity profiles $u_{g}\left(x_{1}\right)$ for different parameters calculated at depth of $35 \mathrm{~nm}$ from interface source/bicrystalc
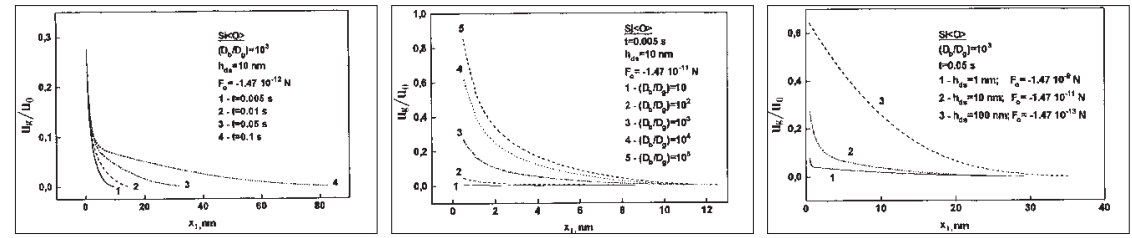

Figure 5. 3. si $\langle O\rangle$ impurity profiles $u_{g}\left(x_{1}\right)$ for different parameters calculated at depth of $35 \mathrm{~nm}$ from interface source/bicrystalc

different distances from interface bicrystal/source. An example of such $3 D$ picture with two $2 D$ cross-sections for the pair $G e\langle S b\rangle$ is given in Fig.2. The influence of parameters, defining diffusion of impurities in a bicrystal, on the character of impurity profiles $u_{g}\left(x_{1}\right)$ in the vicinity of GB is studied below. Figs. 3 and 4 present the results of computer simulation for pairs $G e\left\langle S b>\right.$ and $S i<A l>$ with $F_{0}>0$. As is seen from Fig. 3a, when diffusion time is increasing, a diffusant $(S b)$, coming some distance along GB "core", begins to penetrate into the grain. When the impurity arrives into the grain, elastic force $F\left(x_{1}\right)$ (rapidly diminishing with distance $x_{1}$ by the exponential law) repels it from GB "core". In consequence of this repulsion, the diffusant moves in some region of the grain with a relatively higher velocity than in standard Fisher model. When $F\left(x_{1}\right)$ becomes vanishingly small, impurity atoms will continue to move in the grain-bulk by Fick's laws (as for usual Fisher model). An increase of diffusion velocity in some region of the grain results in the shift of $u_{g}\left(x_{1}\right)$ maximum from the GB "core" (as for usual Fisher model when $F\left(x_{1}\right)=0$ ) into the grain depth. By so doing, the value of $u_{g}\left(x_{1}\right) / u_{0}$ maximum increases in time and the maximum moves deeper and deeper into the grain (Fig. 3a). At constant diffusion time $t$ the value and position of maximum $u_{g}\left(x_{1}\right) / u_{0}$ depends on the ratio $D_{b} / D_{g}$ (Fig. $3 \mathrm{~b}$ ) and density of dislocations at GB "core" (Fig. 3c). Note that for higher dislocation densities (small $h_{d s}$ ) an elastic force may be so strong that maximum $u_{g}\left(x_{1}\right) / u_{0}$ becomes $>1$ (curve 1 in Fig. 3c). This means that accumulation of impurity at all the time is going in only a very narrow region of the grain bulk (where force $F\left(x_{1}\right)$ is a factor). Comparison of results shows that the shape of $u_{g}\left(x_{1}\right)$ profiles for positive $F\left(x_{1}\right)$ is mainly defined by the product $F\left(x_{1}\right) D_{g}$ which determines the proportion of diffusion and drift contributions 
for impurities transfer.Just due to this reason the shift of $u_{g}\left(x_{1}\right) / u_{0}$ maximum deeper into the grain for the pair $S i\langle A l\rangle$ is exhibited only at high $t$ values even for the lowest $h_{d s}$ (see Figs 4a and 4c), as distinct from the behavior of diffusion in $G e<S b>$ bicrystals. This is conditioned by a very small value of the product $F\left(x_{1}\right) D_{g}$ in $S i<A l>$ (by 6 order of magnitude lower than for $G e<S b>)$.

Let us consider the peculiarities of $u_{g}\left(x_{1}\right)$ profiles formation in the pairs of Si $\langle O\rangle$ and $S i<B\rangle$ which are characterized by negative sign of elastic force $\left(F_{0}<0\right)$. This implies that for these two pairs an elastic force will attract impurity to GB "core" preventing the "spreading-out" of impurity around GB, unlike the standard Fisher model. As calculations show, the character of this $u_{g}\left(x_{1}\right)$ "compressing" is mainly dictated by the relationship of drift and diffusion contributions to the impurity transfer. So for $S i\langle B\rangle$ pair (whose $F\left(x_{1}\right) D_{g}$ is nearly by 7 orders smaller than for $S i<O>$ ) the profiles $u_{g}\left(x_{1}\right)$ at moderate densities of dislocations $\left(h_{d s} \sim 10^{-8} \mathrm{~m}\right)$ are merely slightly diverged from the standard Fisher profiles. This implication is derived from the fact that boron easily overcomes the action of elastic force retarding the diffusion "spreading-out" of a diffusant.

Referring to Fig. 5, for $S i\langle O\rangle$ pair a great value of $F\left(x_{1}\right) D_{g}$ offers outstanding distinction of $u_{g}\left(x_{1}\right)$ calculated by a modified Fisher model from the usual Fisher profiles. In this case, although at a relatively small density of dislocations ( $h d s \sim 10^{-7} \mathrm{~m}$ ), a part of diffusant (oxygen) overcomes the force barrier (curve 3 in Fig. 5c) and continues to be transferred in the grain bulk with a high rate (due to high $D_{g}$ ), nevertheless concentration $u_{g}\left(x_{1}\right)$ in the bulk is relatively small even for high values of diffusion time (as for $\mathrm{Si}<$ $B>$ pair). Under these circumstances, $u_{g}\left(x_{1}\right)$ profiles are characterized by a specific bend (Fig. 5a) for long times of diffusion. At the same time, when $h_{d s}$ decreases down to $10^{-9} \mathrm{~m}$, an elastic force of attraction to the dislocation wall drastically increases hindering oxygen escape into the narrow near-boundary layer and into the grain depth. In such a case, $u_{g}\left(x_{1}\right)$ profiles are very similar to the $S i<B>$ ones for comparable $h_{d s}$.

\section{SUMMARY}

Using a modified Fisher model we have shown that elastic stresses which arise due to lattice mismatching of neighboring grains in a bicrystal GB exhibit the radical effect on impurity transfer in the vicinity of GB. For a positive elastic force $F\left(x_{1}\right)$, which rapidly diminishes with distance $x_{1}$ by the exponential law, impurity repelled from GB "core" and maximum of profile $u_{g}\left(x_{1}\right)$ shifted from GB "core" (as for the standard Fisher's model) into the grain bulk. The value and position of impurity profile therewith are defined both by the time of diffusion and the product $F\left(x_{1}\right) D_{g}$. At the determined values of $F\left(x_{1}\right) D_{g}$ the impurity concentration in a very narrow region of the grain can exceed its value co in the source, that is favored by impurity segregation which is very often displayed in the experiments. By contrast, a negative elastic force 
$F\left(x_{1}\right)$ results in "compression" of standard Fisher profiles conserving $u_{g}\left(x_{1}\right)$ maximum at the GB "core".

\section{REFERENCES}

[1] I. Kaur and W. Gust. Fundamentals of Grain and Interphase Boundary Diffusion. Ziegler Press. Stuttgart (1989).

[2] S. Amelincks and W. Dekeyser. The Structure and Properties of Grain Boundaries, In: Solid State Physics. Ed. by F. Seitz and D. Turnbul. Academic press. New York (1959). P. 362.

[3] Samarskii A.A. Theory of difference schemes. Nauka, Moscow, 1977 (in Russian).

[4] Samarskii A.A., Vabishchevich P.N., Matus P.P. // Differential equations. No 2 (1996), P. $265-274$.

[5] Samarskii A.A., Vabishchevich P.N., Matus P.P. // Dokl. ANB, No 5 (1996), P. 9 - 14.

[6] Rychagov V.G. // Preprint IM ASB. No 8(520), (1996), P. 11.

[7] D. Shaw Atomic Diffusion in Semiconductors. Plenum press, London (1973), Chapter

[8] G. V.Samsonov Handbook of Physical and Chemical Properties of Elements. Naukova Dumka, Kiev (1965), (in Russian).

[9] A. Fedotov, A. Drozdovski and S. Shumski. // Polycrystalline Semiconductors IVPhysics, Chemistry and Technology. Ed. by S. Pizzini, H. P. Strunk and J. H. Werner, Solid State Phenomena, Vols. 51-52 (1996), P. 33. 